\title{
Magnetization of a superconducting film in a perpendicular magnetic field
}

\author{
Mauro M. Doria, ${ }^{1,2, *}$ Ernst Helmut Brandt, ${ }^{1,3}$ and F. M. Peeters ${ }^{1}$ \\ ${ }^{1}$ Departement Fysica, Universiteit Antwerpen, Groenenborgerlaan 171, B-2020 Antwerpen, Belgium \\ ${ }^{2}$ Instituto de Física, Universidade Federal do Rio de Janeiro, 21941-972, Rio de Janeiro, Rio de Janeiro, Brazil \\ ${ }^{3}$ Max-Planck-Institut für Metallforschung, Heisenbergstr. 1, D-70569 Stuttgart, Germany
}

(Received 11 March 2008; published 7 August 2008)

\begin{abstract}
With large thin superconducting films in a perpendicular magnetic field, the usual definition and calculation of the magnetization $M$ via currents or as the difference of two fields fail, since the spatially averaged magnetic field in the film coincides with the uniform applied field and the demagnetization factor is unity. The definition of $M$ as field-derivative of the free energy, however, still works in this limit. We generalize the virial theorem, previously derived for infinite bulk superconductors, to infinitely extended films of arbitrary thickness. An expression for $M$ is obtained that indeed reproduces the $M$ computed from the field derivative of the free energy.
\end{abstract}

DOI: 10.1103/PhysRevB.78.054407

PACS number(s): 74.25.Ha, 74.25.Dw, 74.78.Na, 74.50. $+\mathrm{r}$

\section{INTRODUCTION}

A standard theoretical method to obtain the magnetic moment $\mathbf{m}$ of a conductor or of a superconductor induced by the presence of an external applied magnetic field $\mathbf{H}_{a}\left(\mathbf{B}_{a}\right.$ $\left.\equiv \mu_{0} \mathbf{H}_{a}\right)$ is to calculate the integral below over the specimen volume $V$,

$$
\mathbf{m}=\frac{1}{2} \int_{V} d^{3} r \mathbf{r} \times \mathbf{j},
$$

where $\mathbf{j}(\mathbf{r})$ is the current density. Far away from the specimen the currents yield the magnetic induction $\mathbf{B}(\mathbf{r})=\nabla \times \mathbf{A}$ $\left(\mathbf{A}=\mu_{0} \mathbf{m} \times \mathbf{r} / r^{3}\right)$, which renders the magnetic moment detectable by the mechanical torque $\mathbf{m} \times \mathbf{B}_{a}$ and by the force $(\mathbf{m} \cdot \nabla) \mathbf{B}_{a}=\nabla\left(\mathbf{m} \cdot \mathbf{B}_{a}\right)$ (since $\left.\nabla \cdot \mathbf{B}_{a}=0\right)$ exerted in case $\mathbf{B}_{a}$ is inhomogeneous. ${ }^{1}$ In addition, these currents partly screen the specimen interior from the applied field $\mathbf{H}_{a}$. However these currents depend on how the conducting electrons react to $\mathbf{H}_{a}$. In normal conductors, currents exist only in time-dependent $\mathbf{H}_{a}$ and lead, e.g., to the skin effect in high frequency alternating $\mathbf{H}_{a}$ and to a $\mathbf{m}$ that exponentially decays in time after a dc field $\mathbf{H}_{a}$ is switched on or off. Distinctively the induced currents may persist even when the $\mathbf{H}_{a}$ is held constant or is switched off in superconductors. Moreover in this case there are two kinds of static supercurrents: Meissner screening currents that flow in a surface layer of depth $\approx \lambda$ (the London penetration depth) and (or) currents circulating around Abrikosov $^{2}$ vortices. All these currents superimpose linearly to a good approximation if the vortex cores are well separated; in this case the London theory applies. ${ }^{3,4}$ In the general case, the supercurrents and their magnetic field may be calculated from the Ginzburg Landau (GL) theory that is valid also when the cores overlap strongly at high magnetic fields. Hereafter we use the notation $\mathbf{B}(\mathbf{r})$ to describe the local magnetic induction and $\mathbf{B} \equiv\langle\mathbf{B}(\mathbf{r})\rangle$ for the average magnetic induction.

In this paper we use the GL theory to compute the spatially averaged magnetization $\mathbf{M}=\mathbf{m} / V$ of a superconducting film subject to an external applied field perpendicular to its surface. However Eq. (1) relies on a finite-volume integration, whose meaning for infinite specimens must be better defined because of surface (Meissner) currents and its contribution in comparison to the internal (Abrikosov vortices) currents. For instance, the theoretical treatment of bulk superconductor does not take into account external surfaces, but experimentally, bulk superconductors are just those of size $\gg \lambda$. Hence the theoretical treatment takes that all internal currents and fields originate only from the presence of Abrikosov vortices and surface (Meissner) current effects are not considered. From the other side thin films of thickness $d<2 \lambda$ may contain currents and magnetic fields even in the absence of vortices, since any point in the film is "close to the surface."

A special case of interest is mesoscopic superconductors $(\leq \lambda)$ that have attracted attention because of their unique properties (vortex shells, ${ }^{5}$ first-order phase transitions, ${ }^{6}$ giant vortices, ${ }^{7}$ and nonquantized magnetic flux ${ }^{8}$ ). The magnetic properties of very thin superconducting disks subject to a perpendicular magnetic field have been experimentally measured for several radii and its magnetic properties theoretically investigated by means of the Ginzburg-Landau theory. ${ }^{9-11}$ In these cases the magnetization was obtained by averaging the local induction $\mathbf{B}(\mathbf{r})$ inside the film. ${ }^{12}$ However, as we shall see below, it is not possible to do so in case of the infinitely extended film (or films with large aspect ratio) because then this average just gives the applied external field $\mathbf{H}_{a}$.

To better understand the vortex contribution to $\mathbf{M}$ we review a few basic facts about vortices in thin films with $d$ $\ll \lambda$. They are called Pearl ${ }^{13}$ vortices. Both Abrikosov's vortex lines and Pearl's point vortices have a normal core, i.e., a tube in which the superconducting order parameter or GL function $\psi$ decreases from its Meissner-state value to zero over a length $\approx \xi$, the superconducting coherence length. Around Abrikosov vortices in the bulk, the magnetic field and the circulating currents extend to a radius $r \approx \lambda$ and for larger $r$ they decay exponentially as $r^{-1 / 2} \exp (-r / \lambda)$ (if $\lambda$ $>2 \xi$, see Ref. 14). Around Pearl vortices the currents decay much more slowly; the thickness-integrated current density (sheet current) is $J(r)=\Phi_{0} /\left(2 \pi \mu_{0} \Lambda r\right)$ for $r \ll \Lambda$ and $J(r)$ $=\Phi_{0} /\left(\pi \mu_{0} r^{2}\right)$ for $r \gg \Lambda$, where $\Lambda=\lambda^{2} / d$ is the twodimensional penetration depth. The theory of vortices in films of arbitrary thickness $d$ is given for isolated London 
vortices in Ref. 15 and for a periodic vortex lattice from GL theory in Ref. 16. In the half space $z>0$ above the planar surface of films of arbitrary thickness, the magnetic stray field of a single vortex approximately looks like the threedimensional (3D) radial field of a magnetic monopole of strength $2 \Phi_{0}$ positioned at a depth $z \approx-1.27 \lambda$ (if the film is thick enough). ${ }^{15} \mathrm{~A}$ vortex lattice with spacing $a$ shows a periodic stray field whose amplitude decreases rapidly as $\exp (-2 \pi z / a)$ away from the planar surface. ${ }^{16}$

For superconductors of any shape, the magnetic moment $\mathbf{m}$ induced by an applied field $\mathbf{H}_{a}$ is dominated by the term stemming from the Meissner screening currents. This negative (diamagnetic) term is partly compensated by the positive (paramagnetic) term generated by the currents of the vortices. For superconductors of finite sizes, this argument may be used to compute $\mathbf{m}$, but for infinitely extended superconductors it fails since $\mathbf{M}$ becomes the difference of two infinite terms.

Thus, one has to obtain $\mathbf{M}$ from other methods, not from Eq. (1). These methods are the thermodynamic definition of m and the virial expression, which are both able to directly compute this difference of the two infinite terms. The former is the derivative of the free energy while the latter is based on the sum of kinetic and field-free energy contributions with different weights. In this paper we obtain a virial expression for the film under a perpendicular applied field using scaling properties of the GL theory and show that these two methods yield exactly the same result for $\mathbf{M}$. The connection of the virial theorem to scaling properties has been studied by many in the literature. ${ }^{17-25}$

\section{INFINITE SPECIMENS}

Here we review a few examples where the use of Eq. (1) presents difficulties to obtain M. Equation (1) is used to obtain $\mathbf{m}$ in many geometries, e.g., a thin disk such that the $\mathbf{B}(\mathbf{r})$ distribution is known inside. ${ }^{26}$ However one has to be cautious when taking the limit of infinite extension since typically a great part of the magnetic moment comes from the screening currents that flow at the surface. A simple but still surprising example is a thin rectangular strip in a perpendicular magnetic field along $z$. From Eq. (1) one then has $\mathbf{m}=\hat{\mathbf{z}} m, m=\frac{1}{2} \int d x \int d y\left(x J_{y}-y J_{x}\right)$, where $\mathbf{J}(x, y)=\left(J_{x}, J_{y}\right)$ is the sheet current circulating in the strip. In the limit of infinite length along $y$, usually the currents in the strip are assumed to flow all in $y$ direction, and thus the term $y J_{x}$ in the integral is omitted. This is wrong, however, since the two terms in the integral give the same contribution to $m$, irrespective of the strip length, as follows from $\operatorname{div} \mathbf{J}=0$. Thus, in the limit of long strip length $L$ the magnetic moment of the strip is $m$ $=L \int d x x J_{y}(x)$, without a factor $\frac{1}{2}$, since the $\mathrm{U}$ turn of the current at the two far-away ends of the strip contributes $\frac{1}{2}$ of $m .^{27-30}$

Similarly, for a long cylinder of length $d$ in a parallel field $\mathbf{H}_{a}$ that causes a vortex lattice, in the London limit the contribution of each Abrikosov vortex to $m$ is $d \Phi_{0} / \mu_{0}$, as can be shown by direct integration. The volume integral (1) would thus yield the wrong result $m=V B / \mu_{0}$ or $M=m / V=B / \mu_{0}$, where $B$ is the induction or flux density. The correct result is, however, $M=B / \mu_{0}-H_{a}$, which even has opposite sign and originates by accounting for the circulating surface currents.

For the infinite film considered in this paper, the situation with the general definition (1) is even worse. We show this in the thin-film limit using the London approach. From the above formula for the sheet current of a Pearl vortex, $J(r$ $\gg \Lambda)=\Phi_{0} /\left(\pi r^{2} \mu_{0}\right)$, Eq. (1) yields for the magnetic moment of a single Pearl vortex centered in a circular film of radius $R \gg \Lambda$ the large value $m_{v}=\Phi_{0} R / \mu_{0}$, which diverges linearly with the film radius but, interestingly, does not depend on the film thickness $d$ and penetration depth $\Lambda$. This seemingly strange result can be understood as follows: Consider a long thin superconductor strip with half width $w \gg \lambda$ or $w \gg \Lambda$ $=\lambda^{2} / d$ if the thickness $d<\lambda$ is small and with length $L \gg w$. An applied perpendicular field $\mathbf{H}_{a}$ induces in this ideally diamagnetic strip the sheet current $J_{y}(x)=-2 x H_{a} /\left(w^{2}-x^{2}\right)^{1 / 2}$ that flows over the entire strip width, not only at the two edges. ${ }^{27-30}$ The magnetic field of this sheet current has the $z$ component $-H_{a}$ inside the strip, and thus the total field there has zero perpendicular component, i.e., this current screens the interior of the strip from the applied field. The $x$ component of the field inside the strip is finite, $H_{x}(x, z)$ $=J_{y}(x) \sinh (z / \lambda) / 2 \sinh (d / 2 \lambda)$, but this is irrelevant in our context; note that this parallel field at the upper and lower surfaces differs by the sheet current $H_{x}(x, d / 2)-H_{x}(x$, $-d / 2)=J_{y}(x)$ as it should be. This sheet current $J_{y}(x)$ generates a magnetic moment $m=L \int_{-w}^{w} d x J_{y}(x) x=\pi w^{2} L H_{a}$ perpendicular to the strip (the missing factor $\frac{1}{2}$ is discussed above). Now allow vortices to penetrate the film such that screening almost vanishes and the magnetic moment is nearly zero. The induction of these Pearl vortices should thus be $B$ $\approx \mu_{0} H_{a}$ and their number $2 w L B / \Phi_{0}$. Each of these Pearl vortices, therefore, has to carry a magnetic moment $m_{v}$ $=\pi w^{2} L H_{a} \Phi_{0} /(2 w L B) \approx \pi w \Phi_{0} / \mu_{0}$. This result has to be compared with the above magnetic moment of a Pearl vortex in a circular disk $m_{v}=\Phi_{0} R / \mu_{0}$. It indeed agrees if one puts m.

\section{DEMAGNETIZATION EFFECTS}

In this section we briefly review the demagnetization effects in view of the peculiar properties of the superconducting film with large aspect ratio $w / d \gg 1$. In the particular case of a homogeneous superconductor with the shape of an ellipsoid, containing either no vortices (Meissner state) or an ideal vortex lattice in which vortex pinning can be disregarded, demagnetization effects may be described by a demagnetization factor $N$ with $0 \leq N \leq 1$. This is so since in this case a homogeneous applied field $\mathbf{H}_{a}$ causes a demagnetizing field that inside the specimen is homogeneous and superimposes to $\mathbf{H}_{a}$ such that it reduces the internal field by compensating it partly. ${ }^{31}$ For arbitrary orientation of $\mathbf{H}_{a}$ this demagnetization is described by a demagnetization tensor $\stackrel{\leftrightarrow}{N}$ such that the effective external field inside the superconductor is

$$
\mathbf{H}_{\mathrm{eff}}=\mathbf{H}_{a}-\stackrel{\leftrightarrow}{N} \mathbf{M}
$$

When $\mathbf{H}_{a}$ is along one of the three principal axes of the ellipsoid, the tensor reduces to one of its (scalar) diagonal 
terms $N_{1}, N_{2}$, and $N_{3}$, which satisfy $N_{1}+N_{2}+N_{3}=1$. From this sum rule one immediately concludes that $N=1 / 3$ for the sphere, $N=1 / 2$ for long cylinders in a transverse $\mathbf{H}_{a}$, and $N=0$ in parallel $\mathbf{H}_{a}$, and for large flat superconductors such as thin plates or films one has $N=0$ in parallel $\mathbf{H}_{a}$ and $N$ $=1$ in perpendicular $\mathbf{H}_{a}$, this last case being the one of interests here.

As an example consider a superconducting ellipsoid with one axis along $z$ and $\mathbf{H}_{a}=\hat{\mathbf{z}} H_{a}$ along $z$ such that $\mathbf{H}_{a}=\hat{\mathbf{z}} H_{a}$, $\mathbf{H}_{\text {eff }}=\hat{\mathbf{z}} H_{\text {eff }}$, and $\mathbf{M}=\hat{\mathbf{z}} M$. To get further insight let us consider the following approximation: Inside the ellipsoid the constitutive law (or magnetization curve) of the vortex lattice is the same as in the bulk, i.e., surface effects and the periodic stray field outside the surface are disregarded, in contrast to the film example considered below. This local magnetization curve for the bulk $(N=0)$ may be calculated, e.g., from GL theory $^{32}$ as $M\left(H_{a} ; N=0\right)=B / \mu_{0}-H_{a}$ where $B$ is the flux density or induction. The homogeneous magnetization of this ellipsoid follows then from the implicit equation ${ }^{31}$

$$
H_{\text {eff }}=H_{a}-N M\left(H_{\text {eff }} ; N=0\right)
$$

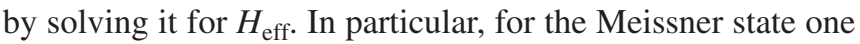
has zero internal field $B=0$, thus $M\left(H_{a} ; 0\right)=-H_{a}$. Inserting this into Eq. (3) we obtain $H_{\mathrm{eff}}=H_{a}-N \cdot\left(-H_{\mathrm{eff}}\right)$, thus (1 $-N) H_{\text {eff }}=H_{a}$ or

$$
M=M\left(H_{a} ; N\right)=M\left(H_{\mathrm{eff}} ; 0\right)=-H_{\mathrm{eff}}=\frac{-H_{a}}{1-N} .
$$

This Meissner state exists in an increasing applied field as long as $0<H_{\text {eff }}<H_{c 1}\left(H_{c 1}\right.$ is the lower critical field) equivalent to applied fields $0<H_{a}<H_{c 1}^{\prime}=(1-N) H_{c 1}$. Thus, for parallel geometry $(N=0)$ one has $H_{c 1}^{\prime}=H_{c 1}$, but for thin films $(1-N \ll 1)$ the magnetic field penetrates at a much lower field $H_{c 1}^{\prime} \ll H_{c 1}$.

In the limit of an infinitely extended film $(N=1)$ one has $H_{c 1}^{\prime}=0$, i.e., even a very small applied field $H_{a}$ penetrates; the average internal field or induction always equals the applied field, $B=\mu_{0} H_{a}$; and the above described definition of the magnetization cannot be used since $N \rightarrow 1$ and the denominator in Eq. (4) vanishes.

\section{THERMODYNAMIC DEFINITION}

In this section we review the thermodynamic method to obtain the magnetization $\mathbf{M}=\mathbf{m} / V$. For the infinite film containing a periodic vortex lattice this method does not require knowledge of the screening currents that may flow, e.g., near the film edges and strongly depend on the shape of the film. ${ }^{33}$ One only has to know the free energy of the ideally periodic vortex lattice solution.

The applied field $\mathbf{B}_{a}=\mu_{0} \mathbf{H}_{a}$, changed by an infinitesimal amount $\delta \mathbf{B}_{a}$, performs the work $\delta F=\mathbf{m} \delta \mathbf{B}_{a}$. We select $z$ as the direction perpendicular to the film surface such that $\mathbf{B}$ $=\mathbf{B}_{a}=\mu_{0} \mathbf{H}_{a}$ and $\mathbf{M}$ are both along $z$ as in Ref. 16. We obtain

$$
M=-\frac{\partial(F / V)}{\partial B} .
$$

$F$ is the Helmholtz free energy of the film which is a function of the average induction or vortex density, previously defined as $\mathbf{B}=\langle\mathbf{B}(\mathbf{r})\rangle$. We define the deviation from the average value

$$
\mathbf{b}(\mathbf{r})=\mathbf{B}(\mathbf{r})-\mathbf{B}=\left[b_{x}(\mathbf{r}), b_{y}(\mathbf{r}), b_{z}(\mathbf{r})\right] .
$$

The free-energy density of a planar film of arbitrary thickness $d$ (its usual value minus $B^{2} / \mu_{0}$ ) averaged over the film or any vortex cell within GL theory reads

$$
\frac{F}{V}=\left\langle\frac{|\mathbf{D} \psi|^{2}}{2 m}+\alpha|\psi|^{2}+\frac{\beta}{2}|\psi|^{4}\right\rangle_{1}+\left\langle\frac{b^{2}}{2 \mu_{0}}\right\rangle_{2},
$$

where $\alpha<0$ and $\beta>0$ are the usual GL coefficients and D $=(\hbar / i) \nabla-2 e \mathbf{A}$. The brackets mean the 3D averages

$$
\langle\ldots\rangle_{1}=\int_{-d / 2}^{d / 2} \frac{d z}{d}\langle\ldots\rangle_{S}, \quad\langle\ldots\rangle_{2}=\int_{-\infty}^{\infty} \frac{d z}{d}\langle\ldots\rangle_{S},
$$

where $S=\Phi_{0} / B$ is the unit-cell area of the vortex lattice. We also define the outer average

$$
\langle\ldots\rangle_{3}=\langle\ldots\rangle_{2}-\langle\ldots\rangle_{1}=2 \int_{d / 2}^{\infty} \frac{d z}{d}\langle\ldots\rangle_{S} .
$$

The last term in Eq. (7) contains both the energy of the magnetic field inside the film (minus $B^{2} / \mu_{0}=\mu_{0} H_{a}^{2}$ ) and the stray-field energy $F_{\text {stray }}$ of the periodic field variations outside the film. The periodic GL solution $\omega(\mathbf{r})=|\psi|^{2}$ and $\mathbf{Q}(\mathbf{r})=\mathbf{A}-(\hbar / 2 e) \nabla \varphi \quad[$ the gauge-invariant supervelocity, with $\varphi$ the phase of $\psi=\sqrt{\omega} \exp (i \varphi)]$ for the film may be computed as described in Ref. 16. When expressed in reduced units the free energy $F / V$, Eq. (7) coincides with the $F_{\text {tot }}$, Eq. (7) of Ref. 16.

\section{SCALING}

In this section we review the virial theorem ${ }^{34}$ for the GL superconductor that relates the sum of the kinetic energy plus twice the field energy to the product of the induction $\mathbf{B}$ times the equilibrium applied field $\mathbf{H}_{a}$ :

$$
\mathbf{B} \cdot \mathbf{H}_{a}=\int_{V} \frac{d^{3} x}{V}\left[\frac{|\mathbf{D} \psi|^{2}}{2 m}+\frac{B(\mathbf{r})^{2}}{\mu_{0}}\right] .
$$

This relation holds for bulk superconductors, that is, for a superconductor that borders with itself due to periodic boundary conditions. From it the magnetization is obtained rendering the same result of the thermodynamic derivative of Eq. (5).

This virial relation is derived by a scaling transformation applied simultaneously to the three coordinates $(x \rightarrow \lambda x, y$ $\rightarrow \lambda y$, and $z \rightarrow \lambda z)$. The Gibbs free energy $G=F-\mathbf{B} \cdot \mathbf{H}_{a}$ is postulated to be extremal under this transformation $\partial G(\lambda) /\left.\partial \lambda\right|_{\lambda=1}=0$. This elegant argument has been extended to microscopic theory in the context of the Eilenberger equations. ${ }^{35}$ In the case when $\mathbf{H}_{a}$ and $\mathbf{B}$ are aligned it yields an explicit relation for $H_{a}(B)$, useful for determining the properties of the vortex lattice ${ }^{36}$

$$
H_{a}=\left\langle\frac{|\mathbf{D} \psi|^{2}}{2 m}+\frac{B(\mathbf{r})^{2}}{\mu_{0}}\right\rangle_{V} / B
$$

that can be expressed in terms of $M=B / \mu_{0}-H_{a}$ : 


$$
-M=\left\langle\frac{|\mathbf{D} \psi|^{2}}{2 m}+\frac{\mathbf{b}(\mathbf{r})^{2}}{\mu_{0}}\right\rangle_{V} / B .
$$

Notice that Eq. (11) contains the field energy, whereas the square of the deviation from the magnetic induction enters Eq. (12). It is possible to obtain the three components of $\mathbf{M}$ independently from such scaling arguments, ${ }^{37}$ an important matter in case of anisotropy. Here we focus on the isotropic situation and in this case the scalar product $\mathbf{M} \cdot \mathbf{B}$ is sufficient to determine $\mathbf{M}$ as a function of $\mathbf{B}$ since both vectors are oriented along the same direction. Formula (12) can also be regarded from another perspective, namely, of obtaining the kinetic energy ${ }^{38}$ as a function of the magnetization and the induction since these two quantities are experimentally accessible. Besides the product $-M B$ is the kinetic energy as long as the local induction $\mathbf{B}(\mathbf{r})$ does not deviate much from its spatial average $\mathbf{B}$. Indeed the last term in formula (12) can be disregarded because the variance of the magnetic field of the vortex lattice $\left\langle\mathbf{b}(\mathbf{r})^{2}\right\rangle$ is very small if the GL parameter $\kappa=\lambda / \xi$ is large or $B$ is close to the upper critical field $B_{c 2}$ $=\mu_{0} H_{c 2}=\Phi_{0} /\left(2 \pi \xi^{2}\right)$; one has ${ }^{32}$ at not too large $B / B_{c 2}$ a constant variance $\left\langle\mathbf{b}(\mathbf{r})^{2}\right\rangle \approx 0.0037 \Phi_{0}^{2} / \lambda^{4}$ and in general $\left\langle\mathbf{b}(\mathbf{r})^{2}\right\rangle$ $\approx B_{c 2}^{2}\left(1-B / B_{c 2}\right)^{2} / 7 \kappa^{4} \ll 1$.

\section{VIRIAL THEOREM FOR FILMS}

In this section we derive a virial relation for the superconducting film. Scaling invariance just along the film is applied, which means that the coordinates parallel to the film are scaled but not the perpendicular one $(x \rightarrow \lambda x, y \rightarrow \lambda y$, and $z \rightarrow z$ ). In this way one obtains the following virial relation for the magnetization of an infinite film, first with arbitrary orientation of $\mathbf{H}_{a}$ :

$$
-\left(\frac{1}{2} \mathbf{M}_{\|}+\mathbf{M}_{z}\right) \cdot \mathbf{B}=\left\langle\frac{\left|\mathbf{D}_{\|} \psi\right|^{2}}{2 m}\right\rangle_{1}+\left\langle\frac{b_{\|}^{2}+2 b_{z}^{2}}{2 \mu_{0}}\right\rangle_{2},
$$

with $\mathbf{M}_{\|}=\mathbf{M}_{x}+\mathbf{M}_{y}, \mathbf{D}_{\|}=\mathbf{D}_{x}+\mathbf{D}_{y}$, and $b_{\|}^{2}=b_{x}^{2}+b_{y}^{2} ; \mathbf{b}(\mathbf{r})$ is defined in Eq. (6). If $\mathbf{H}_{a}$ is along $z$, and thus also $\mathbf{B}$ and $\mathbf{M}$, Eq. (13) reduces to

$$
-M=\left[\left\langle\frac{\left|\mathbf{D}_{\|} \psi\right|^{2}}{2 m}\right\rangle_{1}+\left\langle\frac{b_{\|}^{2}+2 b_{z}^{2}}{2 \mu_{0}}\right\rangle_{2}\right] / B .
$$

The distinct contributions to the magnetization can be treated separately through this formula, which is the sum of the kinetic and two other magnetic terms associated to the energy inside and outside the film:

$$
\begin{gathered}
-M_{1}=\left\langle\frac{\left|\mathbf{D}_{\|} \psi\right|^{2}}{2 m}\right\rangle_{1} B, \\
-M_{2}=\left\langle\frac{b_{\|}^{2}+2 b_{z}^{2}}{2 \mu_{0}}\right\rangle_{1} B, \\
-M_{3}=\left\langle\frac{b_{\|}^{2}+2 b_{z}^{2}}{2 \mu_{0}}\right\rangle_{3} / B, \quad \text { and } \\
M=M_{1}+M_{2}+M_{3} .
\end{gathered}
$$

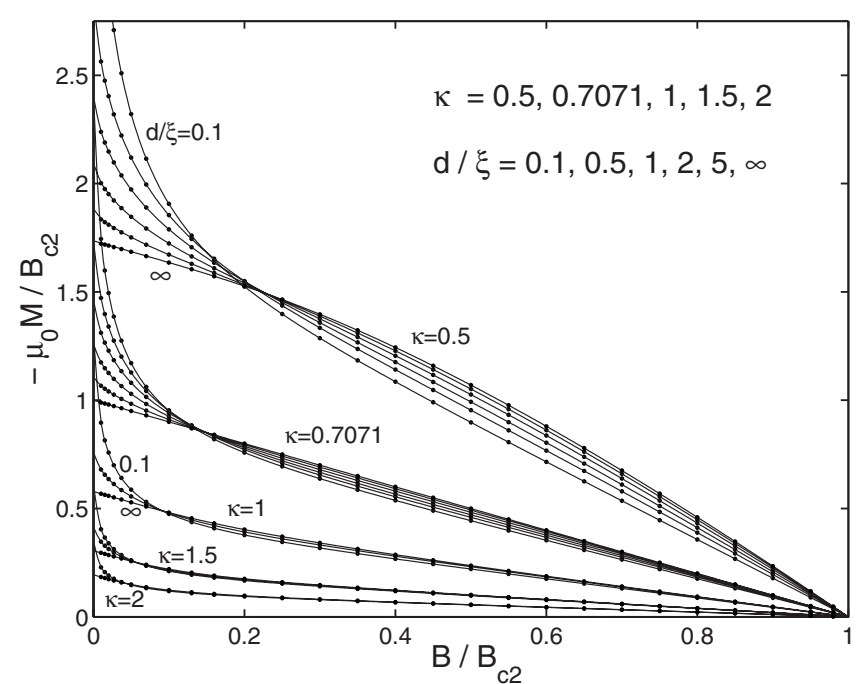

FIG. 1. The magnetization $M$ of a superconducting infinite film in a perpendicular magnetic field $B_{a}=B$ for film thicknesses $d / \xi$ $=0.1,0.5,1.2,5$, and $\infty$, and GL parameters $\kappa=0.5,1 / \sqrt{2}, 1,1.5$, and 2 . The solid lines are calculated from the energy-derivative Eq. (5) and the dots are from the virial expression Eq. (13), rendering excellent agreement. In the bulk limit $d \rightarrow \infty$ at $B \rightarrow 0, \mu_{0} M(B)$ reaches the value $B_{c 1}$ and has the slope -1 .

Below we shall use Eq. (14) to compute the magnetization $M(B)$ and compare it with that obtained from the energy derivative Eq. (5). The contribution from outside the film is related to the stray-field energy $F_{\text {stray }}$ whose energy according to Ref. 16 is

$$
F_{\text {stray }} / d=\left\langle b_{x}^{2}+b_{y}^{2}+b_{z}^{2}\right\rangle_{3} / 2 \mu_{0} .
$$

The periodic stray field above a planar surface satisfies the Laplace equation $\nabla^{2} \mathbf{b}(\mathbf{r})=0$; from this one can show that $\left\langle b_{x}^{2}+b_{y}^{2}\right\rangle_{3}=\left\langle b_{z}^{2}\right\rangle_{3}$. This relation directly yields for the second term in Eq. (14) the outer contribution

$$
\left\langle b_{x}^{2}+b_{y}^{2}+2 b_{z}^{2}\right\rangle_{3} / 2 \mu_{0}=\frac{3}{2} F_{\text {stray }} / d .
$$

\section{NUMERICAL COMPARISON}

To compare the $M$ values of Eqs. (5) and (14) we use the numerical method of Ref. 16, where the trial functions for the periodic functions $|\psi(x, y, z)|^{2}, \mathbf{B}(x, y, z)$, and the supervelocity $\mathbf{Q}=\mathbf{A}-(\hbar / 2 e) \nabla \varphi$, are finite Fourier series. Then we minimize the free energy with respect to the Fourier coefficients by means of iteration equations. Within this numerical method the free energy decreases smoothly until it becomes stationary with accuracy $10^{-14}$ after $25-50$ iteration steps. For our infinite ideal vortex lattice we choose a triangular symmetry. We are then left with three input parameters: GL parameter $\kappa=\lambda / \xi$, average induction $B=\mu_{0} H_{a}$, and film thickness $d$. We do this computation, e.g., for $B / B_{c 2}$ $=0.007,0.01,0.015,0.02,0.027, \ldots, 0.9,0.95,0.98$ and obtain the derivative $\partial F(B) / \partial B$ by numerical interpolation of $F(B)$. Figure 1 shows the resulting magnetization versus $B$ in units of $B_{c 2}$ for several values $\kappa=0.5,1 / \sqrt{2}, 1,1.5,2$, and for 


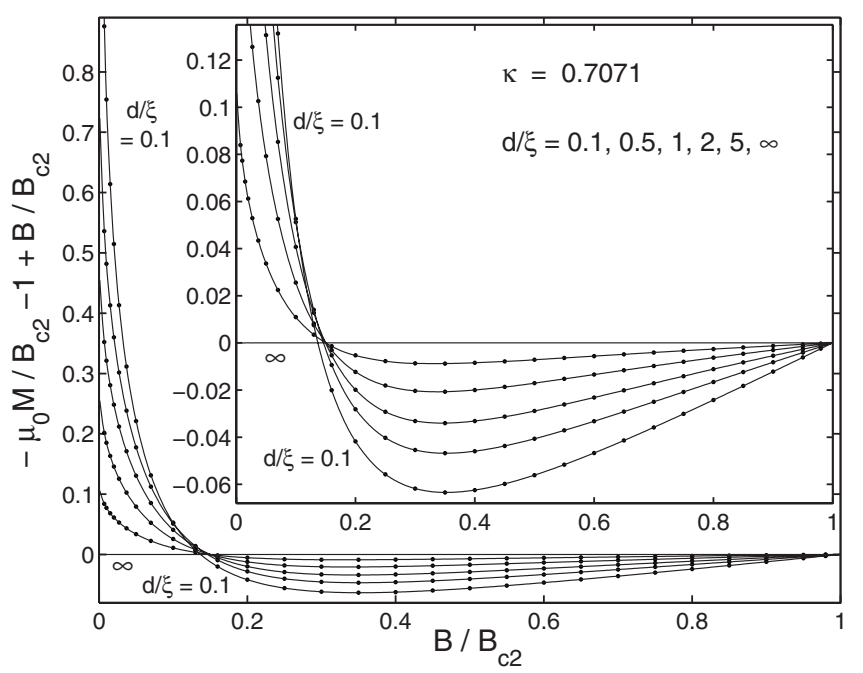

FIG. 2. The same magnetization $M$ as in Fig. 1 but only for GL parameter $\kappa=1 / \sqrt{2}$, plotted with reference to the bulk result, represented by the $d \rightarrow \infty$ horizontal line: $-\mu_{0} M / B_{c 2}-1+B / B_{c 2}$. This large scale plot shows the excellent agreement between the dots (virial expression) with the lines (energy derivative) also in the high $B$ region.

thicknesses $d / \xi=0.1,0.5,1,2,5, \infty$. The solid lines show the $M=-\partial(F / V) / \partial B$ and the dots show the virial expression for $M$, Eq. (14). One can see that all the dots are on top of the lines, meaning that the virial argument yields the correct magnetization both for thin and thick films.

The special case $\kappa=1 / \sqrt{2}$ is particularly useful for checking the precision of our numerics, since then in the bulk limit $(d \gg \lambda, \xi)$ one has $F / V=-\frac{1}{2}\left(1-B^{2} / B_{c 2}^{2}\right)$, and thus $-\mu_{0} M / B_{c 2}=1-B / B_{c 2}$ is a straight line. This is indeed confirmed by our numerics to high precision. Figure 2 uses this feature to plot the $M$ for $\kappa=1 / \sqrt{2}$ in the form $-\mu_{0} M / B_{c 2}$ $-1+B / B_{c 2}$, which yields a horizontal line when $d \rightarrow \infty$. This Fig. 2 clearly shows the perfect agreement between the two theoretical methods to obtain $M$ in the entire range $0<B$ $<B_{c 2}$.

For larger $\kappa>1 / \sqrt{2}$ the thick-film curve $-M(B)$ is concave, i.e., it has positive curvature. For smaller $\kappa<1 / \sqrt{2}$ (type-I superconductors) $-M(B)$ is convex for thick films, which means the vortex lattice is unstable. However, for sufficiently thin films, even for $\kappa<1 / \sqrt{2}$ the curvature of $-M(B)$ can be positive and may change sign at a certain value of $B$. Indeed one can show that thin films of type-I superconductors can contain a stable vortex lattice and thus behave like type-II superconductors.

The curves $-M(B)$ for various thicknesses $d$ cut each other in the same neighborhood, near the field $B^{*} \approx B_{c 2} / \kappa$ $=\Phi_{0} /\left(2 \pi \lambda^{2}\right)$, i.e., for this $B$ value $M$ is almost independent of the film thickness. Thus for a fixed $B>B^{*}$ the magnetization increases with $d$ while it decreases for $B<B^{*}$. Such peculiar properties are special features of the vortex state in large thin superconducting films.

The following are useful exact results found for the film: For thick films the initial slope is $-\left.M^{\prime}(B)\right|_{B=0}=-1$ independently of $\kappa$, as confirmed by Fig. $1 .-M(0)=H_{c 1}$, where the lower critical field with good accuracy for all $\kappa$ is ${ }^{32}$

$$
\begin{gathered}
B_{c 1}=\mu_{0} H_{c 1}=\frac{\Phi_{0}}{4 \pi \lambda^{2}}[\ln \kappa+\alpha(\kappa)], \\
\alpha(\kappa) \approx \frac{1}{2}+\frac{1+\ln 2}{2 \kappa-\sqrt{2}+2} .
\end{gathered}
$$

This is so since when surface contributions to the energy may be disregarded, one has for superconducting ellipsoids $-M=H_{c 1}$ at $H_{a}=H_{c 1}^{\prime}=(1-N) H_{c 1}$, where $B=0$, cf. Eq. (4).

Note that in Figs. 1 and 2 that for thin films, $-M(B)$ exhibits a sharp upturn at low inductions $B \ll B_{c 2}$, such that for $d / \xi=0.1$, and $-M$ is enhanced by more than a factor of 2 as compared to thick films or the bulk. This enhancement stems from the energy of the magnetic stray field that enhances the self-energy of a vortex beyond its bulk value of $d \Phi_{0} H_{c 1}$. This enhancement of the stray field on $-M(B)$ may also be brought out by plotting the various terms in the virial expression, Eq. (15), separately. Figure 3 shows features of the three contributions to $M$. One can see that for thin films both $M_{1}$ and $M_{3}$ exhibit a sharp increase as $B \rightarrow 0$, and the stray-field contribution $M_{3}$ yields a considerable contribution to $M$. This is so since at low $B$ the vortex spacing becomes larger than $\Lambda$ and thus the periodic variation of the stray field becomes large, while at higher $B$ the vortex fields overlap strongly and this variation is small. In the depicted cases of small $\kappa$ the contribution of $M_{2}$ is negligibly small for thin films but for thick films it is a considerable fraction of $M$ at low $B$. However, for larger $\kappa$ and at higher fields $B$, this $M_{2}$ contribution is negligibly small even on thick films since it decreases quadratically as $\left(1-B / B_{c 2}\right)^{2} / \kappa^{2}$, while the main term $M_{1}$, and thus the total $M$, decreases only linearly as $\left(1-B / B_{c 2}\right) / \kappa$. The variation of the inner magnetic field may thus be safely disregarded in practically all cases except for small $\kappa$ and $B$ in thick films or bulk superconductors. See Ref. 16 for a detailed discussion of the amplitudes and energies of the (outer) stray field and the "inner stray field," i.e., the field variation inside the film but near the surface.

Finally, Fig. 4 shows the product $M B$ plotted versus $B$ for $\kappa=0.71,1,1.5$, and three film thicknesses. Due to the assumed large extension of the film (large aspect ratio $w / d$ $\gg 1$ ) one has $B=H_{a}$, thus the plot also may be interpreted as showing $M H_{a}$ versus $H_{a}$. According to the above discussion following Eq. (12) these parabolalike plots show the kinetic energy $M_{1} B$ to a good approximation. The small deviation $\left(M_{2}+M_{3}\right) B$ may be seen from Fig. 3 .

\section{CONCLUSION}

We have shown that the magnetization $\mathbf{M}$ of superconducting films with large aspect ratio $w / d \gg 1$ can be computed either by the usual thermodynamic energy derivative, Eq. (5), or by a virial expression obtained here by scaling arguments, Eq. (14). Notice that for such films the spatially averaged inner field equals the (perpendicular) applied field $B=\langle B(\mathbf{r})\rangle=B_{a}=\mu_{0} H_{a}$. We numerically show here for a large range of parameters $B, \kappa$, and $d$ that both methods yield the same $M$.

We have pointed out here that the basic definition of $\mathbf{m}$ as an integral over the circulating currents does not work for 

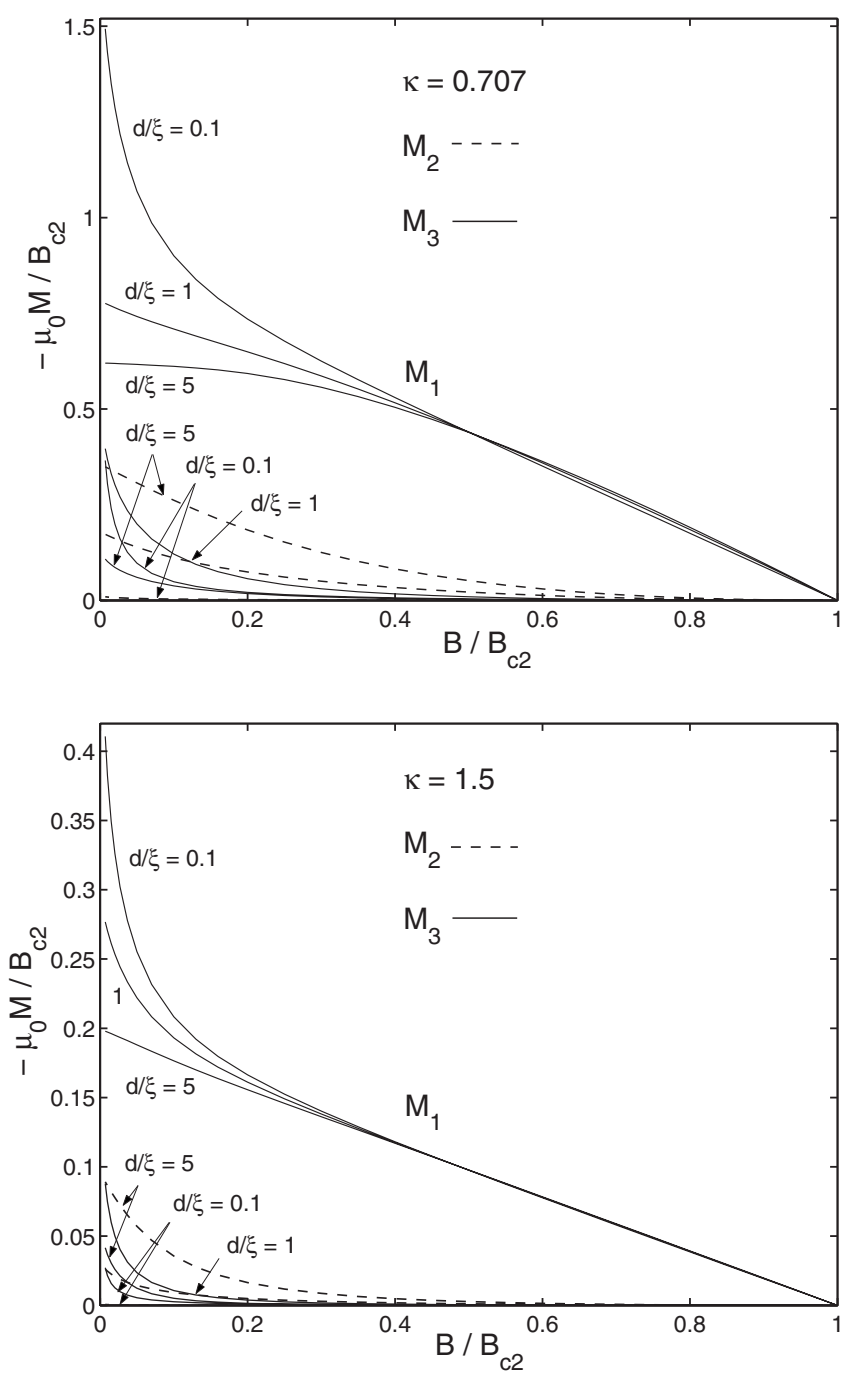

FIG. 3. The virial terms that contribute to the magnetization, Eq. (13), are shown here for $\kappa=1 / \sqrt{2}$ (top) and $\kappa=1.5$ (bottom) and three values of the film thicknesses $d=0.1 \xi, \xi, 5 \xi$. While the kineticenergy term $M_{1}$ is monotonically increasing with decreasing thickness $d$, the magnetic-field terms $M_{2}$ (from inside the film) and $M_{3}$ (from outside) are nonmonotonic functions of $d$. Both the stray field $M_{3}$ and the main term $M_{1}$ exhibit a sharp increase for thin films as $B \rightarrow 0$. The inner field energy $M_{2}$ is considerable for thick films and small $B$ for the depicted cases of small $\kappa$, but it is negligibly small for thin films and always for large $\kappa$ and/or large $B$.

infinite films since the screening currents that flow over the entire film are not known in this case because they depend on the shape of the film. As opposed to this, the periodic part of the currents (from the vortex lattice) is well known. Interestingly, these screening currents (analogous to the surface currents of a long cylinder in parallel field) and the detailed shape of the large film do not enter the other two definitions of its magnetic moment, since they depend only on the periodic parts of the current, field, and order parameter.

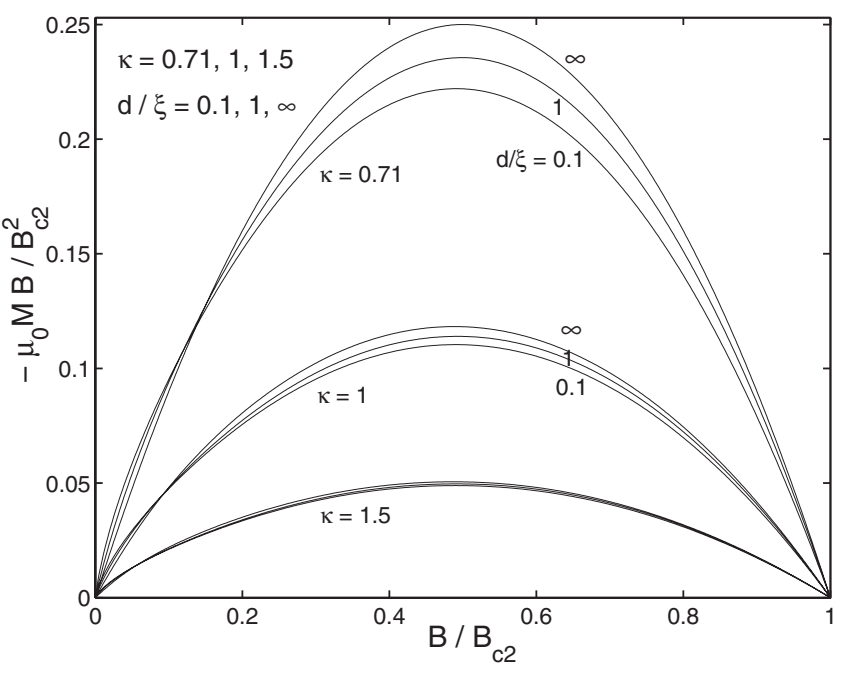

FIG. 4. The product $M B$ as a function of $B=\mu_{0} H_{a}$ for $\kappa$ $=0.71,1,1.5$ and film thicknesses $d / \xi=0.1,1, \infty$. Since $M=M_{1}$ $+M_{2}+M_{3}$ with typically $M_{2}+M_{3} \ll M_{1}$, cf. Fig. 3, the plotted quantity to a good approximation shows the kinetic energy $M_{1} B$.

We have extended for films the bulk result that the kinetic energy of the condensate can be directly retrieved from the reversible equilibrium magnetization $M$ (Ref. 38) by means of the virial relation ${ }^{34}$ in case of a not too small $\kappa$. The virial relation for films encourages one to apply the vortex-shaking method to measure the reversible magnetic moment in realistic, not pin-free superconducting films, and then obtain the kinetic energy. Luckily, for flat film superconductors even in the presence of vortex pinning the reversible perpendicular magnetization can be measured. This is done by applying an additional small ac magnetic field in the film plane ${ }^{39}$ that forces the irreversible currents to relax. This intricate vortex shaking effect has been explained for ac field transverse to ${ }^{40}$ and parallel to ${ }^{41}$ the screening currents in a thin strip, as well as for the circulating currents in a rectangular film ${ }^{42}$ and also for tilted applied dc field. ${ }^{43}$ Recently the kinetic-energy density of low- $T_{c} \quad(\mathrm{Nb})$ and high- $T_{c} \quad\left(\mathrm{YBa}_{2} \mathrm{Cu}_{3} \mathrm{O}_{7-x}\right.$ and $\left.\mathrm{Bi}_{2} \mathrm{Sr}_{2} \mathrm{CaCu}_{2} \mathrm{O}_{8+\delta}\right)$ compounds was obtained from reversible magnetization measurements showing very distinct behavior in the neighborhood of the critical temperature $T_{c}{ }^{44}$ The present results suggest that such study be also done in films made of low and high $T_{c}$ compounds. As shown here, in this case too, the kinetic-energy density can be read from magnetization measurements by means of the virial theorem for not too small $\kappa$ superconductors.

\section{ACKNOWLEDGMENTS}

Part of this work was supported by the Flemish Science Foundation (FWO-Vl), the ESF-AQDJJ network, and the bilateral project between Flanders and Brazil. M.M. Doria also thanks CNPq, FAPERJ, and BOF/UA (Belgium). 
*mmd@if.ufrj.br

${ }^{1}$ W. Greiner, Classical Electrodynamics (Springer, Berlin, 1998).

${ }^{2}$ A. A. Abrikosov, Zh. Eksp. Teor. Fiz. 32, 1442 (1957)[Sov. Phys. JETP 5, 1174 (1957)].

${ }^{3}$ M. Tinkham, Introduction to Superconductivity (McGraw-Hill, New York, 1966).

${ }^{4}$ E. H. Brandt, Rep. Prog. Phys. 58, 1465 (1995).

${ }^{5}$ I. V. Grigorieva, W. Escoffier, J. Richardson, L. Y. Vinnikov, S. Dubonos, and V. Oboznov, Phys. Rev. Lett. 96, 077005 (2006).

${ }^{6}$ A. K. Geim, I. V. Grigorieva, S. V. Dubonos, J. G. S. Lok, J. C. Maan, A. E. Filippov, and F. M. Peeters, Nature (London) 390, 259 (1997).

${ }^{7}$ A. Kanda, B. J. Baelus, F. M. Peeters, K. Kadowaki, and Y. Ootuka, Phys. Rev. Lett. 93, 257002 (2004).

${ }^{8}$ A. K. Geim, S. V. Dubonos, I. V. Grigorieva, K. S. Novoselov, F. M. Peeters, and V. A. Schweigert, Nature (London) 407, 55 (2000).

${ }^{9}$ P. S. Deo, V. A. Schweigert, F. M. Peeters, and A. K. Geim, Phys. Rev. Lett. 79, 4653 (1997).

${ }^{10}$ P. S. Deo, V. A. Schweigert, and F. M. Peeters, Phys. Rev. B 59, 6039 (1999).

${ }^{11}$ V. A. Schweigert, F. M. Peeters, and P. S. Deo, Phys. Rev. Lett. 81, 2783 (1998).

${ }^{12}$ V. A. Schweigert and F. M. Peeters, Phys. Rev. B 57, 13817 (1998).

${ }^{13}$ J. Pearl, Appl. Phys. Lett. 5, 65 (1964).

${ }^{14}$ L. Kramer, Phys. Rev. B 11, 3821 (1971).

${ }^{15}$ G. Carneiro and E. H. Brandt, Phys. Rev. B 61, 6370 (2000).

${ }^{16}$ E. H. Brandt, Phys. Rev. B 71, 014521 (2005).

${ }^{17}$ N. G. Van Kampen, Rep. Math. Phys. 3, 235 (1972).

${ }^{18}$ C. Von Westenholtz, Ann. Inst. Henri Poincare, Sect. A 29, 415 (1978).

${ }^{19}$ M. Brack, Phys. Rev. D 27, 1950 (1983).

${ }^{20}$ E. Papp, Phys. Rep. 136, 103 (1986).

${ }^{21}$ B. Nachtergaele and A. Verbeure, J. Geom. Phys. 3, 315 (1986).
${ }^{22}$ N. Kimura and K. Sogo, Prog. Theor. Phys. 78, 72 (1987).

${ }^{23}$ A. E. Jacobs and L. Benguigui, Phys. Rev. A 39, 3622 (1989).

${ }^{24}$ W. Lucha and F. F. Schöberl, Phys. Rev. Lett. 64, 2733 (1990).

${ }^{25}$ E. A. Dudas and D. Pirjol, Phys. Lett. B 260, 186 (1991).

${ }^{26}$ M. Nakagawa, S. Utsumi, and Y. Oda, Phys. Rev. B 60, 1372 (1999).

${ }^{27}$ E. H. Brandt, Phys. Rev. B 54, 4246 (1996).

${ }^{28}$ E. H. Brandt, M. V. Indenbom, and A. Forkl, Europhys. Lett. 22, 735 (1993).

${ }^{29}$ E. H. Brandt, Phys. Rev. B 49, 9024 (1994).

${ }^{30}$ E. H. Brandt and M. V. Indenbom, Phys. Rev. B 48, 12893 (1993).

${ }^{31}$ E. H. Brandt, Phys. Rev. B 60, 11939 (1999).

${ }^{32}$ E. H. Brandt, Phys. Rev. B 68, 054506 (2003).

${ }^{33}$ E. H. Brandt, Phys. Rev. B 72, 024529 (2005).

${ }^{34}$ M. M. Doria, J. E. Gubernatis, and D. Rainer, Phys. Rev. B 39, 9573 (1989).

${ }^{35}$ U. Klein and B. Pöttinger, Phys. Rev. B 44, 7704 (1991).

${ }^{36}$ E. H. Brandt, Phys. Rev. Lett. 78, 2208 (1997).

${ }^{37}$ Mauro M. Doria and S. C. B. de Andrade, Phys. Rev. B 53, 3440 (1996).

${ }^{38}$ Mauro M. Doria, S. Salem-Sugui, I. G. de Oliveira, L. Ghivelder, and E. H. Brandt, Phys. Rev. B 65, 144509 (2002).

${ }^{39}$ M. Willemin, C. Rossel, J. Hofer, H. Keller, A. Erb, and E. Walker, Phys. Rev. B 58, R5940 (1998).

${ }^{40}$ G. P. Mikitik and E. H. Brandt, Phys. Rev. Lett. 89, 259701 (2002).

${ }^{41}$ G. P. Mikitik and E. H. Brandt, Phys. Rev. B 67, 104511 (2003).

${ }^{42}$ G. P. Mikitik and E. H. Brandt, Phys. Rev. B 69, 134521 (2004).

${ }^{43}$ E. H. Brandt and G. P. Mikitik, Supercond. Sci. Technol. 20, S111 (2007).

${ }^{44}$ S. Salem-Sugui, Jr., Mauro M. Doria, A. D. Alvarenga, V. N. Vieira, P. F. Farinas, and J. P. Sinnecker, Phys. Rev. B 76, 132502 (2007) 